\title{
OPTIMIZATION OF 5G VIRTUAL-CELl BASED COORDINATED MULTIPOINT NETWORKS USING DEEP MACHINE LEARNING
}

\author{
Mohamed Elkourdi, Asim Mazin and Richard D. Gitlin \\ Department of Electrical Engineering, University of South Florida \\ Tampa, Florida 33620, USA
}

\begin{abstract}
Providing seamless mobility and a uniform user experience, independent of location, is an important challenge for $5 G$ wireless networks. The combination of Coordinated Multipoint (CoMP) networks and Virtual-Cells (VCs) are expected to play an important role in achieving high throughput independent of the mobile's location by mitigating inter-cell interference and enhancing the cell-edge user throughput. Userspecific VCs will distinguish the physical cell from a broader area where the user can roam without the need for handoff, and may communicate with any Base Station (BS) in the VC area. However, this requires rapid decision making for the formation of VCs. In this paper, a novel algorithm based on a form of Recurrent Neural Networks (RNNs) called Gated Recurrent Units (GRUs) is used for predicting the triggering condition for forming VCs via enabling Coordinated Multipoint (CoMP) transmission. Simulation results, show that based on the sequences of Received Signal Strength (RSS) values of different mobile nodes used for training the RNN, the future RSS values from the closest three BSs can be accurately predicted using GRU, which is then used for making proactive decisions on enabling CoMP transmission and forming VCs.
\end{abstract}

\section{KEYWORDS}

Coordinated multipoint (CoMP), machine learning (ML), self-organizing networks (SON), recurrent neural networks (RNN), gated recurrent unit $(G R U)$.

\section{INTRODUCTION}

The fifth generation $(5 \mathrm{G})$ of mobile networks using mmWave technology is anticipated to deliver a substantial increase in the rates of data traffic over the cellular network, as much as $10 \mathrm{Gbps}$ compared to $100 \mathrm{Mbps}$ in 4G networks [1]. For instance, such applications as streaming UltraHigh Definition (UHD) video, Augmented Reality (AR) and Virtual Reality (VR), which have emerged under the 5G Enhanced Mobile Broadband (eMBB) use case, require very high throughput rates everywhere even at the cell edges (i.e., providing a uniform user experience) [1]. However, degradation in the throughput can be witnessed, particularly in cellular systems with a frequency reuse of unity, as the user equipment (UE) moves towards the cell edge due to several factors such as the path loss and the interference from neighboring cells. This degradation in the perceived throughput could greatly undermine the quality of a real- time applications that requires a very high throughput.

Furthermore, in order to maintain the connectivity of a UE with the network, a handover (HO) process takes place by changing the association of the UE to another cell with a better signal quality. During the HO process, the UE might also experience a degradation in the quality of service as a result of the $\mathrm{HO}$ delay. Thus, providing seamless mobility and a reliable quality of

DOI: 10.5121/ijwmn.2018.10401 


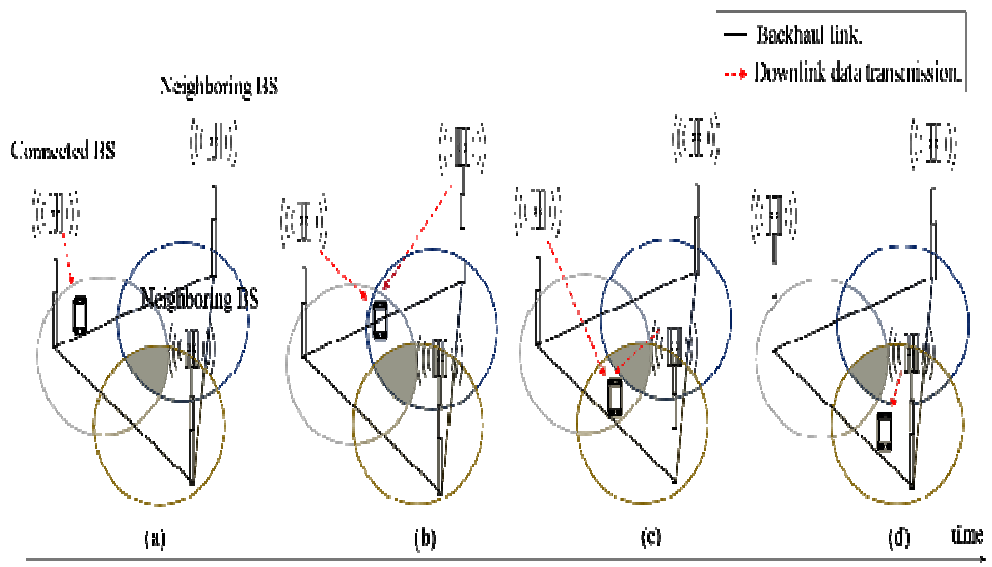

Figure 1. Enabling/ disabling Coordinate Multipoint (CoMP) transmission according to the UE's trajectory. (a) Associating UE with the nearest base station (BS). (b) Forming a Virtual-Cell (VC). (c) Adapting VC according to the UE's mobility. (d) Dissolving VC.

service anywhere in the network for mobile users is an important challenge in 5G cellular networks.

Many solutions have been proposed in the 3GPP standards, such as using self-organizing networks (SON) in order to enhance the cell-edge user throughput via mitigating the inter-cell interference. For instance, an Inter-Cell Interference Coordination (ICIC) technique [2] proposed in Long Term Evolution (LTE), Release 8, and the enhanced ICIC (eICIC) technique proposed [3] in LTE-Advanced (LTE-A), Releases 10 and 11, exploit the interference information exchanged between adjacent cells via the X2 backhaul interface to improve the cell edge user's throughput by adaptively muting resources that cause strong interference and assigning different frequencies and powers for the cell edge users. On the other hand, these techniques can result of a degradation in the overall system throughput due to restricting the usage of radio resources in time and frequency.

Coordinated multipoint (CoMP) transmission [4] is another proposed solution that is also based on cooperation among cells. However, unlike the previous techniques where the UE communicates with only one base station, CoMP can allow cell edge users to communicate with multiple BSs (forming a CoMP cooperating set) and hence improve the throughput of cell edge users and the overall network. CoMP was first standardized in Long Term Evolution-Advanced (LTE-A), Releases 11 and 12.

Moreover, 5G systems are envisioned to further leverage CoMP technology for providing a uniform user experience anywhere in the network. In contrast to the cell-centric approach used in traditional cellular networks with fixed cell coverage areas, a new trend of a user-centric approach, where the UE is associated with multiple BSs and creates a Virtual-Cell (VC) [5] that is adapted according to the mobility of UEs. For instance, using joint transmission-CoMP (JTCoMP) in formation of VCs in the downlink (DL) can help to attain a uniform user experience for mobile users via improving the throughput of cell-edge users and minimizing the number of hard handovers. However, to enable the formation of DL VCs, the UE's data must always be available within BSs in the CoMP cooperating set, leading to a substantial cooperation cost due to the exchange of data and the control information among the cooperating BSs. On the other hand, waiting for CoMP transmission to be enabled can lead to degradation in throughput since the time required to form VCs is severely time constrained. 
International Journal of Wireless \& Mobile Networks (IJWMN) Vol. 10, No. 4, August 2018

The main contribution of this paper is to propose a novel algorithm for proactively predicting the optimal triggering conditions that can be used to dynamically enable/disable CoMP transmission (or to form/dissolve VCs) (see Figure 1) instead of relying on passive approaches. The proposed algorithm is based on using Recurrent Neural Networks (RNNs) with a Gated Recurrent Units (GRUs) mechanism. Given the data about sequences of Received Signal Strength (RSS) values of different mobile nodes for training the GRU, simulation results show that the future RSS values can be rapidly predicted and that, based on user mobility, CoMP transmission can be enabled/disabled with a very high accuracy. One major challenge in order to make a reliable prediction is to collect a sufficient number of sequences in the target areas, so-called "big data".

The remainder of this paper is organized as follows. Section 2 gives an overview of about stateof-the-art using Machine Learning (ML) in SONs. Section 3 represents the use of Recurrent Neural Networks (RNNs) based on a Gated Recurrent Unit (GRU) mechanism for CoMP-DL. Simulation results are discussed in Section 4. Finally, the paper is concluded within Section 5.

\section{Prior State-Of-Art On Using Machine Learning in 5G Self- ORGANIZED NETWORKS}

Machine learning has previously been proposed for SONs. ML for mobility prediction is utilized for making optimal HO decisions in wireless networks. In [6], Luo et al. used a Nonlinear Autoregressive Exogenous Model (NARX) based Neural Network (NN) for handover prediction. The RSS values and the delays between Access Points (APs) are fed to an NN with 12 hidden layers. The NN was able to predict a point which was close to the optimal HO location. Javed et al. [7] used signal strength variation, the past HO rate, number of cells with RSSI values above a certain threshold (active set) and active set update rate as features for the AdaBoost algorithm with Decision Stumps to predict the occurrence of HO. Liou and Huang [8] used the past three location coordinates as features for a Neural Location Indicator for predicting the node's mobility and inferring HOs. Anagnostopoulos et al. [9] used the last four previous cell IDs a user had visited, in four-element features vectors, for predicting the user's next cell ID. They have compared the performance of k-Nearest Neighbors $(\mathrm{kNN})$, Naïve Bayes, Bagging and a vote of kNN, J48 Decision Trees, rule-based learner (JRip) and AdaBoost. In [10], Anagnostopoulos et al. incorporated transition time slots into feature vectors. However, the obtained accuracy on the next cell predictions was very similar to [9]. However, none of the above- proposed ML techniques consider a mobile node to be concurrently associated with multiple base stations (BSs) in creating VCs. In [11], Wickramasuriya et al. used sequences of Received Signal Strength (RSS) values as features for training a Recurrent Neural Network (RNN) classifier based on Long Short-Term Memory (LSTM) to predict the next base station (BS) a mobile node will be associated and the optimal VC topology according to the mobility of users. They did not consider predicting the optimal triggering conditions for enabling/disabling CoMP transmission based on the mobility of users - which is the goal of our work. 


\section{Coordinated Multipoint Transmission Management Based ON RECURRENT NEURAL NETWORKS}

\subsection{SySTEM MODEL}

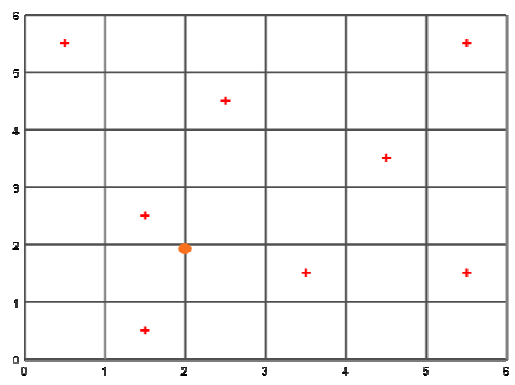

Figure 2. A road network with eight base stations (BSs) (crosses) and a mobile node (circle). The grid lines are the roads. Each mobile node measures the received signal strength (RSS) from the three closest BSs.

A road network of dimensions $6 \mathrm{~km} \times 6 \mathrm{~km}$ with a regularly spaced intersections of $1 \mathrm{~km}$ apart is generated, as shown in Figure 2. Within this grid, Eight BSs are placed in arbitrary locations. A mobile node is randomly generated on a road with an initial speed drawn from a uniform distribution between $8-12 \mathrm{~km} / \mathrm{h}$ for pedestrians and between $55-65 \mathrm{~km} / \mathrm{h}$ for vehicles. It is assumed that a mobile node continues to maintain a constant speed until leaving the road network. At any intersection a mobile node is assigned a probability of 0.5 for moving straight ahead, or an equal probability of 0.25 for turning either right or left. When a node is within the road network, it measures the RSS values from the closest three BSs. We chose this method to demonstrate the capability of RNNs to learn sequences in order to predict RSS values to proactively enable/disable CoMP transmission. The path loss model adopted in the system model, is suggested by 3GPP [12], with an additional term to account for large- scale shadow fading. Here, $\sigma$ is normally distributed with mean zero and variance $9 \mathrm{~dB}$ and $d$ is measured in kilometers.

$$
P L(d)=128.1+37.6 \log (d)+\sigma,
$$

\subsection{DATASET}

An eight-element feature vector is created, where the positions correspond to BSs with their RSS values. The RSS values are recorded every $500 \mathrm{~ms}$ from the closest three BSs to the mobile node. The rest of the vector elements are set to zero. As the mobile node moves along the road network and the set of closest three BSs changes, the RSS values (feature vectors) are recorded and stored consecutively in a queue data structure as follow.

$$
\text { sequence }(\mathrm{i})=\left[\left[\mathrm{RSS}_{1}, \mathrm{RSS}_{2}, \ldots, \mathrm{RSS}_{8}\right],\left[\mathrm{RSS}_{1}, \mathrm{RSS}_{2}, \ldots, \mathrm{RSS}_{8}\right], \ldots,\left[\mathrm{RSS}_{1}, \mathrm{RSS}_{2}, \ldots, \mathrm{RSS}_{8}\right]\right]
$$

Since the recorded RSS values from the distant past do not have a large predictive value regarding the direction the mobile node is heading to, the oldest elements are dropped from the queue when its size exceeds 150 . With this method we simulated mobile nodes until we collected a total of 100,000 sequences.

\subsection{RecurRent Neural Networks (RNN) BASEd on Gated RECURRENT Unit (GRU)}

The RNN is used to discover patterns in sequential information (or temporal data). Unlike traditional neural networks where all inputs (and outputs) are assumed to be independent of each 
other, RNNs have a memory which captures information about what happened in all previous time steps to help learn a large range of dependencies. The GRU was first introduced by Cho et al. [13] as a modification to the hidden layer $(\mathrm{H}) \mathrm{RNN}$, to solve the vanishing gradient problem. The RNN architecture tends to give priority for the most recent inputs and neglects the effects of inputs that are further away in the past, as illustrated in Figure 3.

A GRU is made up of two gates, as shown in Figure 4. The first is the update gate, which controls how much of the current cell content should be updated with the new candidate state. The second is the reset gate, which resets the memory of the cell if it is closed i.e. the unit acts as if the next processed input was the first in the sequence. The state equations of the GRU are [13].

$$
\begin{aligned}
& \text { reset gate }: \boldsymbol{r}[t]=\sigma\left(\boldsymbol{W}_{r} \mathbf{h}[t-1]+\boldsymbol{R}_{r} \boldsymbol{x}[t]+\boldsymbol{b}_{r}\right) \\
& \text { current state }: \mathbf{h}^{\prime}[t]=\mathbf{h}[t-1] \odot \mathbf{r}[t] \\
& \text { candidate state }: \mathbf{z}[t]=g\left(\mathbf{W}_{z} \mathbf{h}^{\prime}[t-1]+\boldsymbol{R}_{z} \mathbf{x}[t]+\boldsymbol{b}_{z}\right), \\
& \text { update gate }: \mathbf{u}[t]=\sigma\left(\mathbf{W}_{u} \mathbf{h}[t-1]+\boldsymbol{R}_{u} \mathbf{x}[t]+\boldsymbol{b}_{u}\right) \\
& \text { new state }: \mathbf{h}[t]=(1-\mathbf{u}[t]) \odot \mathbf{h}[t-1]+\mathbf{u}[t] \odot \mathbf{z}[t]
\end{aligned}
$$

where, $\mathrm{g}($.$) is non-linear function usually implemented by a hyperbolic tangent, \sigma$ is the logistic sigmoid, $\mathbf{W}_{r} \mathbf{W}_{z} \mathbf{W}_{u}$ are rectangular weight matrices, that are applied to the input $\mathrm{x}[\mathrm{t}]$ (RSS values from all eight BSs), $\mathbf{R}_{r} \mathbf{R}_{z} \mathbf{R}_{u}$ are square matrices that define the weights of the recurrent connections, $\mathbf{b}_{r} \mathbf{b}_{z} \mathbf{b}_{u}$ are the bias vectors, and $\odot$ is the Hadamard product.

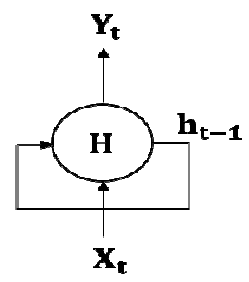

(a)

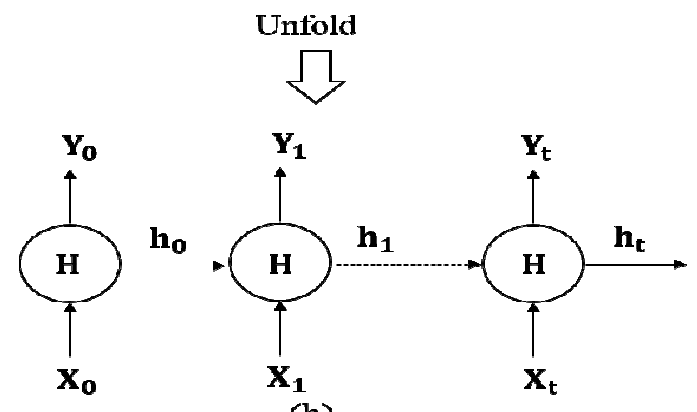

(b)

Figure 3. (a) RNN structure. (b) RNN structure unfolded in time. 


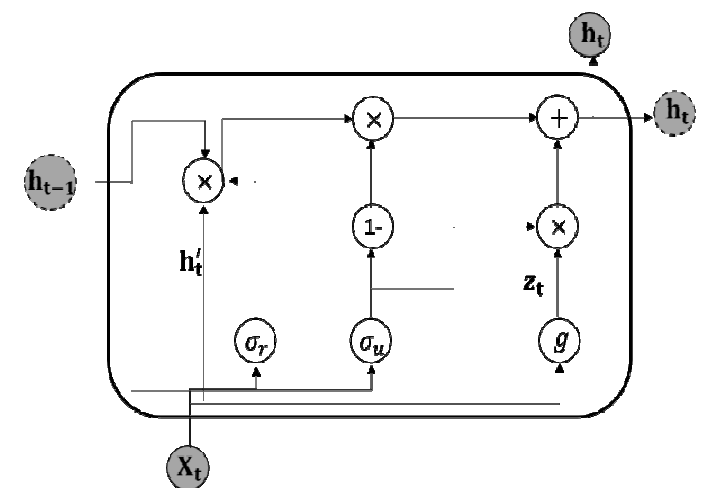

Figure 4. Gated Recurrent Unit (GRU) architecture. Dark gray circles with a solid line are the variables whose content is exchanged with the input and output of the network. Dark gray circles with a dashed line represent the internal state variables, whose content is exchanged within the cells of the hidden layer. White circles with,+ 1 and represent linear operations.

\section{Simulation ReSUltS}

In our simulation, a Gated Recurrent Unit (GRU) mechanism with 512 units is used to predict the RSS values for making a proactive decision on whether to form/dissolve VC via enabling/disabling CoMP transmission (predict the optimal triggering conditions for enabling/disabling CoMP and forming VCs). A total of 70,000 sequences were used for training the GRU model and 30,000 were used for testing it. With 15 training epochs and 75 steps per epoch, the testing error gradually converged. The performance of the GRU model in predicting the RSS values of two different UE from the closet three BSs is shown in Figure 5. It was shown that the GRU model can output predictions on the RSS values that are very close to the true values most of the time. Based on these predictions, the network can proactively make a decision on enabling the CoMP transmission for the goal of providing a uniform user experience.
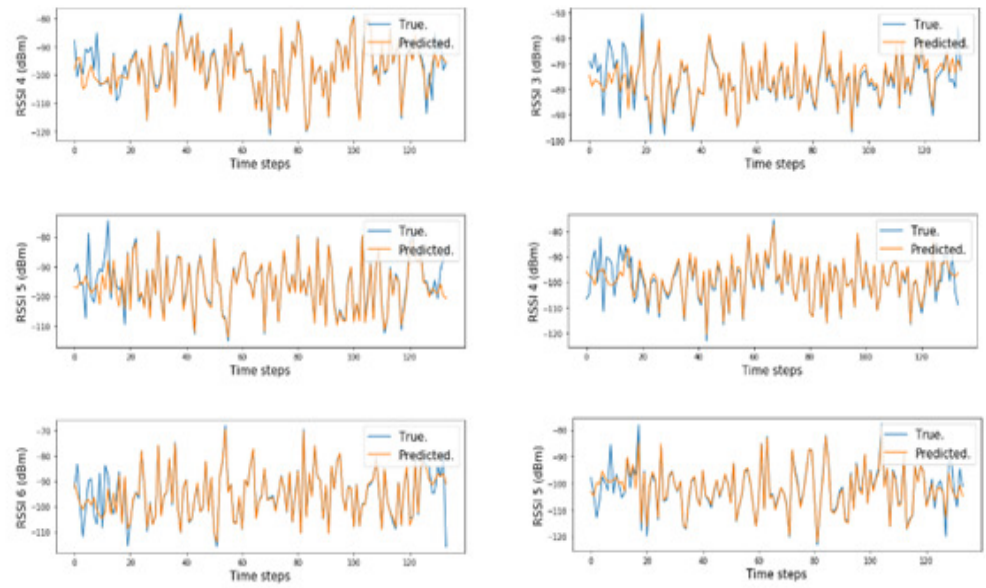

(a)

(b)

Figure 5.True versus predicted Received Signal Strength (RSS) values for two different UE. (a) True and predicted RSS values measured from closet three BSs (4, 5 and 6). (b) True and predicted RSS values measured from closet three BSs (3, 4 and 5).

\section{CONCLUding ReMARKS}

In this paper, the use of deep machine learning for proactive mobility management in $5 \mathrm{G}$ wireless networks is proposed and evaluated in a particular scenario. In particular, proactively predicting 
the triggering conditions to enable/disable CoMP transmission and Virtual-Cell (VC) selection is investigated. A RNN based on GRU mechanism is applied to eight element feature vectors of the RSS values from all the BSs in the network to make an accurate prediction about the RSS values in the future. The proposed algorithm is a promising approach to achieving the goal of providing a uniform user experience everywhere in the network via dynamic formation of VCs. Moreover, considering the very low-latency requirements of next-generation $5 \mathrm{G}$ networks, the proposed algorithm helps the mobility management function facilitate $\mathrm{VC}$ formations.

\section{ACKNOWLEDGEMENTS}

The authors thank Dilranjan S. Wickramasuriya for the fruitful discussions on deep machine learning.

\section{REFERENCES}

[1] J. G. Andrews, S. Buzzi, W. Choi, S. V. Hanly, A. Lozano, A. C. K. Soong, and J. C. Zhang, "What will 5G be?" IEEE Journal on Selected Areas in Communications, vol. 32, no. 6, pp. 1065-1082, June 2014.

[2] J. Zhang and J. G. Andrews, "Adaptive Spatial Intercell Interference Cancellation in Multicell Wireless Networks," in IEEE Journal on Selected Areas in Communications, vol. 28, no. 9, pp. 1455 1468, December 2010. doi: 10.1109/JSAC.2010.101207.

[3] S. Deb, P. Monogioudis, J. Miernik and J. P. Seymour, "Algorithms for Enhanced Inter-Cell Interference Coordination (eICIC) in LTE HetNets," in IEEE/ACM Transactions on Networking, vol. 22, no. 1, pp. 137-150, Feb. 2014.doi: 10.1109/TNET.2013.2246820

[4] H. Dahrouj and Wei Yu, "Coordinated beamforming for the multi-cell multi-antenna wireless system," in Proc. 42nd Annual Conference on Information Sciences and Systems, 2008, pp. 429-434.

[5] J. Kim, H. W. Lee, and S. Chong, "Virtual cell beamforming in cooperative networks," IEEE J. Sel. Areas Commun., vol. 32, no. 6, pp. 1126-1138, 2014.

[6] Y. Luo, P. N. Tran, C. An, J. Eymann, L. Kreft, and A. Timm-Giel, "A novel handover prediction scheme in content centric networking using nonlinear autoregressive exogenous model," in Proc. IEEE Vehicular Technology Conference, 2013, pp. 1-5.

[7] U. Javed, D. Han, R. Caceres, J. Pang, S. Seshan, and A. Varshavsky, "Predicting handoffs in 3G networks," SIGOPS Oper. Syst. Rev., vol. 45, no. 3, pp. 65-70, 2011.

[8] S. Liou and Y. Huang, "Trajectory predictions in mobile networks," International Journal of Information Technology, vol. 11, no. 11, pp. 109- 122, 2005.

[9] T. Anagnostopoulos, C. Anagnostopoulos, S. Haadjiefthymiades, M. Kyriakakos, and A. Kalousis, "Predicting the location of mobile users: a machine learning approach," in Proc. International Conference on Pervasive Services, 2009, pp. 65-72.

[10] T. Anagnostopoulos, C. B. Anagnostopoulos, S. Haadjiefthymiades, A. Kalousis , and M. Kyriakakos, "Path prediction through data mining," in Proc. IEEE International Conference on Pervasive Services, 2007, pp. 128-135.

[11] D. S. Wickramasuriya, C. A. Perumalla, K. Davaslioglu and R. D. Gitlin, "Base station prediction and proactive mobility management in virtual cells using recurrent neural networks," 2017 IEEE 18th Wireless and Microwave Technology Conference (WAMICON), Cocoa Beach, FL, 2017, pp. 1-6.doi: 10.1109/WAMICON.2017.7930254. 
[12] K. Davaslioglu and E. Ayanoglu, "Interference-based cell selection in heterogeneous networks," in Proc. ITA Workshop, San Diego, 2013, pp. 1- 6.

[13] K. Cho, J. Chung, C. Gulcehre, and Y. Bengio, "Empirical evaluation of gated recurrent neural networks on sequence modeling," 2014. [Online]. Available: https://arxiv.org/pdf/1412.3555.

\section{Authors}

Mohamed Elkourdi received the M.Sc. degree in Telecommunication and Signal Processing from New Jersey Institute of Technology in 2013, and the B.Sc. degree in Telecommunication and Electronics Engineering with distinction from Applied Science University, Amman, Jordan in 2010. Currently, he is pursuing his Ph.D. under the supervision of Prof. Richard Gitlin at the University of South Florida with the Innovation in Wireless Information Networking Laboratory (iWINLAB). His research interests include machine learning and game theory in 5G wireless networks and beyond, multiple access techniques, coordinated multipoint (CoMP) and MIMO systems.

Asim Mazin received the B.S. degree in Electronics and Telecommunications engineering in 2007 from the College of Industrial Technology Misurata, Libya and an M.S. degree in Electri-cal and Computer Engineering with a concentration in wireless communications from Southern Illinois University in 2013. Currently, he is pursuing his Ph.D. degree in Electrical Engineering at the University of South Florida with the Innovations in Wireless Information Networking ( $i$ WINLAB) and his research interests include Physical Layer Security, IoT Security, and mmWave MIMO Systems.
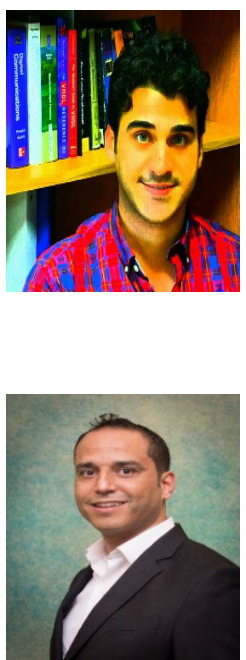

Richard D. Gitlin is a State of Florida 21st Century World Class Scholar, Distinguished University Professor and the Agere Systems Chaired Distinguished Professor of Electrical Engineering at the University of South Florida. He has more than 45 years of leadership in the communications and networking industry. He was at Bell Labs/Lucent Technologies for 32-years performing and leading pioneering research and development in digital communications, broadband networking, and wireless systems. Dr. Gitlin was Senior VP for Communications and Networking Research at Bell Labs and later CTO of Lucent's Data Networking Business Unit.

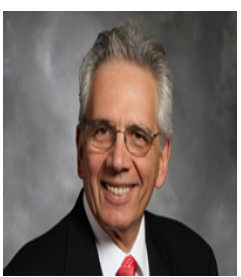
After retiring from Lucent, he was visiting professor of Electrical Engineering at Columbia University, where he supervised several doctoral students and research projects and then Chief Technology Officer of Hammerhead Systems, a venture funded networking company in Silicon Valley. Since joining USF in 2008, he has focused on the intersection of communications with medicine and created an interdisciplinary team that is focused on wireless networking of in vivo miniature wirelessly controlled devices to advance minimally invasive surgery and other cyber-physical health care systems. Dr. Gitlin has also directed research on advancing wireless local and $4 \mathrm{G}$ and $5 \mathrm{G}$ cellular systems by increasing their reliability and capacity. Dr. Gitlin is a member of the National Academy of Engineering (NAE), a Fellow of the IEEE, a Bell Laboratories Fellow, a Charter Fellow of the National Academy of Inventors (NAI), and a member of the Florida Inventors Hall of Fame. He is also a co-recipient of the 2005 Thomas Alva Edison Patent Award and the S.O. Rice prize, has co-authored a text, published $\sim 150$ papers and holds 65 patents. 